\title{
Meibomian gland dysfunction patients with
} novel Sjögren's syndrome biomarkers benefit significantly from a single vectored thermal pulsation procedure: a retrospective analysis

\author{
This article was published in the following Dove Press journal: \\ Clinical Ophthalmology \\ 13 April 2017 \\ Number of times this article has been viewed
}

\author{
Alice T Epitropoulos 1,2 \\ Krysta Goslin² \\ Raman Bedi ${ }^{3}$ \\ Caroline A Blackie ${ }^{4}$ \\ 'Ophthalmic Surgeons and \\ Consultants of Ohio, The Eye Center \\ of Columbus, ${ }^{2}$ The Ohio State \\ University Wexner Medical Center, \\ Department of Ophthalmology, \\ Columbus, OH, USA; ${ }^{3}$ ris Advanced \\ Eye Centre, Chandigarh, India; \\ ${ }^{4}$ TearScience Inc., Morrisville, \\ NC, USA
}

Purpose: To measure the effects from a single vectored thermal pulsation treatment of the meibomian glands on dry eye signs and symptoms in patients who tested positively versus negatively for novel Sjögren's syndrome (SS) biomarkers.

Methods: The retrospective study included the deidentified data of 102 eyes of 59 patients with dry eye and meibomian gland dysfunction (MGD), who were also tested for novel biomarkers for SS and underwent a single 12-minute LipiFlow thermal pulsation procedure. All patients were already being treated with individualized dry eye therapy but remained symptomatic. Meibomian gland secretion (MGS) scores, Standard Patient Evaluation of Eye Dryness (SPEED) questionnaire scores and tear breakup times (TBUTs) before and 8 weeks after thermal pulsation treatment were analyzed.

Results: Twenty-three patients tested positive for novel biomarkers of SS and 36 patients tested negative. At baseline, MGS, SPEED and TBUT of both SS-positive and SS-negative patients were equivalent. At 8 weeks' post-treatment, mean MGS score, SPEED and TBUT

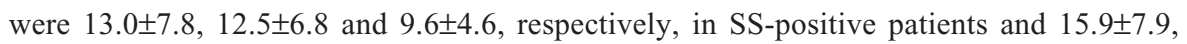
$10.0 \pm 6.3$ and $8.3 \pm 4.6$, respectively, in SS-negative patients $(P<0.001)$. While the posttreatment MGS was significantly better in SS-negative patients than SS-positive $(P=0.021)$, no significant difference between post-treatment SPEED and TBUT was observed between the two groups $(P>0.05)$.

Conclusion: LipiFlow treatment in MGD patients who were SS-positive for novel biomarkers of SS demonstrated improvement in signs and symptoms of dry eye. While improvement in MGS scores in SS-negative patients was higher than that observed in SS-positive patients, SPEED and TBUT were equivalent between these two groups.

Keywords: Sjögren's syndrome, Sjögren's syndrome with MGD, LipiFlow, dry eye

\section{Introduction}

Sjögren's syndrome (SS) is a systemic autoimmune disease characterized by lymphocytic infiltration and dysfunction of the exocrine glands, mainly the salivary and lacrimal glands, resulting in dry mouth and dry eye syndrome due to glandular hyposecretion. ${ }^{1,2}$ Because the pathogenesis of SS involves lacrimal insufficiency, it has conventionally been classified as a major etiology of aqueous tear-deficient dry eye (ATDE). ${ }^{1}$ The ophthalmic treatment of SS remains largely focused on the alleviation of symptoms of ocular dryness using replacement therapy in the form of artificial tear substitutes $^{3,4}$ and/or topical immunomodulators, such as cyclosporine.
Correspondence: Alice T Epitropoulos Ophthalmic Surgeons and Consultants of Ohio, The Eye Center of Columbus, 262 Neil Ave, Columbus, Ohio 43215, USA Tel +l 6148324050

Email aepitrop@columbus.rr.com 
SS patients may manifest signs consistent with meibomian gland dysfunction (MGD) ${ }^{5}$ along with their ATDE and may do so with a degree of severity high enough to considerably worsen ocular surface health. ${ }^{6,7}$ Desiccation stress of the ocular surface and adnexa may explain, in part, why patients with SS manifest MGD. ${ }^{8}$ The increased evaporation of the aqueous component of the precorneal tear film that accompanies MGD-associated lipid layer deficiency may further challenge an already ATDE condition. A review of literature reveals that the meibomian glands of patients with SS were impaired more severely than the glands of dry eye patients without SS. ${ }^{7}$ Evaporation rates were shown to be significantly higher in the ATDEs of SS patients than in patients with non-SS ATDEs. ${ }^{6}$ This concurrence of MGD with ATDE may help to explain why SS patients experience less than optimal improvement in symptom relief using conventional therapy of lacrimal output alone. ${ }^{3}$

As MGD will frequently present as part of the ocular condition of SS patients, optimal long-term management of the ocular surface health of SS patients may involve not only conventional treatment of lacrimal output, such as the use of topical cyclosporine, ${ }^{4}$ but may also now include treatment efforts that target meibomian gland function. To date, there is no report on the benefit of treatments directed toward "rehabilitating" the meibomian glands to improve their function as part of the overall therapeutic approach to restore the health of the ocular surface in SS patients. ${ }^{7,9}$

The core therapeutic approach for the treatment of obstructive MGD is to relieve the obstruction in the meibomian glands. ${ }^{10-12}$ Conventional treatments have included warm compresses for front surface lid warming and lid massage, but do not address obstruction of the meibomian glands. ${ }^{12}$ In the authors' experience, these are supportive therapies that are best offered after obstruction has been addressed. While there is a long history supporting the benefits of forceful manual gland expression, ${ }^{13}$ a vectored thermal pulsation system (LipiFlow, TearScience Inc., Morrisville, NC, USA) is an innovative, automated method, which overcomes the limitations of conventional methods and offers an effective treatment for meibomian gland obstruction. ${ }^{12}$ LipiFlow safely delivers a therapeutic level of heat to the palpebral surfaces of the upper and lower eyelids directly over the meibomian glands, ${ }^{11,14}$ while graded pulsatile pressure simultaneously evacuates the meibomian glands during heating. ${ }^{11,14,15}$ Several studies have shown that a single 12-minute LipiFlow treatment can be effective in relieving obstruction, returning function to meibomian glands, and thereby improving evaporative dry eye (EDE) symptoms. ${ }^{10-12,14,16-19}$ What has not been studied is the effect of LipiFlow thermal pulsation treatment on dry eye in SS, classically described as consisting purely of a deficiency in volume of ATDE. ${ }^{20}$ The purpose of this study is to measure the effects from a single vectored thermal pulsation treatment of the meibomian glands on dry eye signs and symptoms in patients who tested positively versus negatively for novel SS biomarkers with the Sjö test.

\section{Methods}

Deidentified medical records of 76 consecutive patients (119 eyes) who had undergone a single 12-minute LipiFlow procedure for MGD along with a Sjo test because of suspicion of SS (with concurrent dry mouth, dry nose or joint/muscle involvement) were retrospectively reviewed. The procedures were performed between October 2013 and December 2014 at the Eye Center of Columbus, Columbus, $\mathrm{OH}$. The diagnosis of MGD was based on the reduced meibomian gland function as measured with the meibomian gland evaluator (meibomian gland secretion [MGS] score: $0-15 ;{ }^{11}$ see study parameters below for a full description). All patients were already being treated with individualized dry eye treatment (including aqueous and nutritional supplementation and other therapies as needed), but remained symptomatic (Standard Patient Evaluation of Eye Dryness [SPEED] questionnaire) and continued to demonstrate signs and symptoms of dry eye disease.

Of the 76 patients, data of 17 patients (17 eyes) were not available at the postprocedure 8 weeks' follow-up; therefore, a total of 59 patients ( 102 eyes), of which 45 were females and 14 were males with mean age $62 \pm 13.8$ years (range: 19-94 years), were included in the analysis. The study was approved by the ethics committee of the Mount Carmel IRB, Columbus, OH, USA. All research components adhered to the tenets of the Declaration of Helsinki and were conducted in agreement with human research regulations and standards. Patient consent was not required due to the retrospective nature of the study.

Patients were tested for SS using the Sjö test ${ }^{21}$ (Nicox, Bausch and Lomb, Rochester, NY, USA). The Sjö test is a commercially available diagnostic blood test designed to allow for early detection of SS with novel biomarkers (salivary gland protein-1, parotid secretory protein and carbonic anhydrase 6) along with the traditional biomarkers of SS (SS-A/Ro, $\mathrm{SS}-\mathrm{B} / \mathrm{La}$, antinuclear antibody and rheumatoid factor). ${ }^{21}$

\section{LipiFlow device}

The LipiFlow ${ }^{11}$ treatment is indicated in patients with chronic cystic conditions of the eyelids, including MGD. The LipiFlow treatment is contraindicated in patients with coexisting ocular conditions in either eye, which might have 
posed an increased risk of procedure-related injury (eg, active ocular infection or inflammation in either eye); history of ocular surgery or trauma within the last 3 months; ocular surface abnormality potentially compromising corneal integrity in either eye (eg, prior chemical burn, recurrent corneal erosion or corneal epithelial defect); eyelid abnormalities affecting lid function in either eye.

The LipiFlow consists of a disposable ocular component and a nondisposable control system. The disposable component comprises an insulated lid warmer with an eye cup. The lid warmer includes a scleral shell designed to rest on the sclera and vault the cornea. The concave side of the scleral lens is composed of an insulating material, which, in addition to the air gap created by the corneal vault, shields the cornea and ocular surface from exposure to the heat. The convex side of the scleral lens contains an embedded precision heater that heats the palpebral conjunctiva overlying the upper and lower lid meibomian glands. The eye cup contains an inflatable air bladder that rests over the closed eyelids once the scleral lens has been inserted.

The 12-minute therapeutic procedure includes an initial heating phase followed by pulsating pressure, which serves to evacuate any heated softened material from the glands. The bladder inflates and deflates in such a way so as to massage the eyelids from the terminal end of the meibomian gland in the direction of the meibomian gland orifices.

\section{Study parameters}

The outcome measures used to evaluate the effectiveness of the LipiFlow ${ }^{\circledR}$ treatment were the MGS score, subjective dry eye symptoms using SPEED questionnaires and tear breakup time (TBUT).

MGS was standardized using a handheld instrument, Meibomian Gland Evaluator, which applies pressure approximating that of a deliberate blink over the meibomian glands along the distal eyelid, subjacent to the eyelash line. A total of 15 glands were evaluated along the lower eyelid margin, consisting of five consecutive glands located in each of the temporal, central and nasal regions. For each of the 15 glands, characteristics of the expressed gland contents were graded on a scale of $0-3$ where $0=$ no secretion, $1=$ inspissated/filamentary secretion, $2=$ cloudy liquid secretion and $3=$ clear liquid secretion. The total MGS score (range: 0-45) was calculated as the sum of the grades for all 15 glands. ${ }^{11}$

The SPEED ${ }^{22}$ questionnaire assesses the frequency and severity of dry eye symptoms over the last 3 months. The total SPEED score is the sum of the frequency and severity scores for all symptoms over a range from 0 to 28 . A lower score represents less frequent and/or less severe symptoms.
TBUT was performed with fluorescein strip moistened with eye wash solution. The TBUT was measured in seconds with a stopwatch. An average of three readings was used for data analysis.

\section{Statistical analysis}

Statistical analysis was performed using SPSS software (IBM Corp, Somers, NY, USA). Normality of the data was tested using Shapiro-Wilk test. Paired $t$-test (for normally distributed data) and its nonparametric alternative, Wilcoxon rank test, were used to analyze the outcomes and describe the change from baseline to postprocedure at 8 weeks. To compare the post-treatment outcomes between Sjögren'spositive and Sjögren's-negative eyes, an independent $t$-test or its nonparametric alternative, Mann-Whitney $U$ test, was used. A statistically significant difference was based on the level $\alpha=0.05$.

\section{Results}

Twenty-three patients tested positive for novel biomarkers (SS-positive patients) and 36 patients tested negative (SS-negative patients) using the Sjö test. The single 12-minute LipiFlow therapeutic procedure resulted in a significant improvement in signs and symptoms from baseline measurements in both the groups (SS positive and SS negative).

At baseline, all chief parameters, that is, mean MGS, SPEED scores and TBUT scores of both SS-positive and SS-negative patients were equivalent $(P=0.163$ for MGS; $P=0.274$ for SPEED and $P=0.397$ for TBUT). A significant increase in the MGS was observed from pretreatment level to the 8 weeks' post-treatment in both SS-positive $(P<0.001)$ and SS-negative $(P<0.001)$ patients (Figure 1). However,

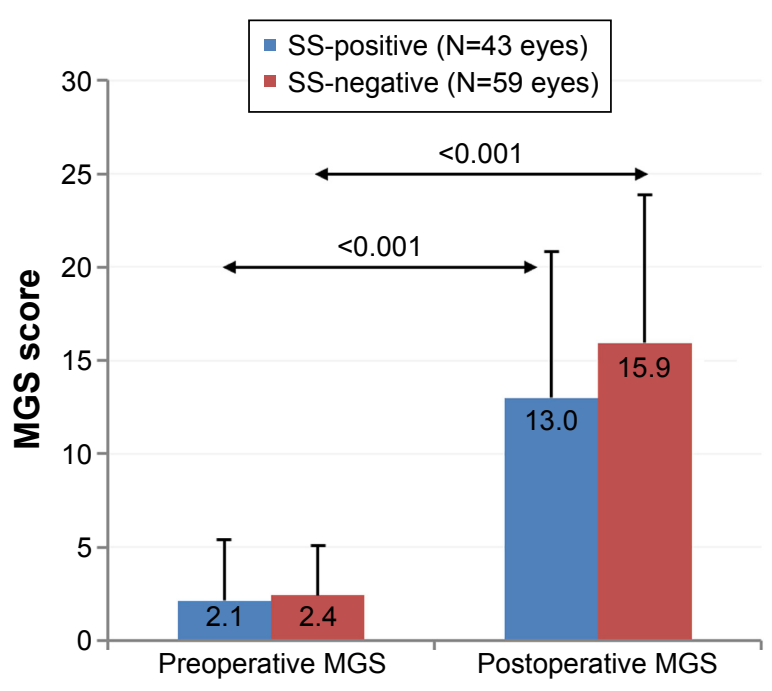

Figure I Effect of LipiFlow treatment on meibomian gland secretion (MGS) score of Sjögren's syndrome (SS)-positive and SS-negative patients. 


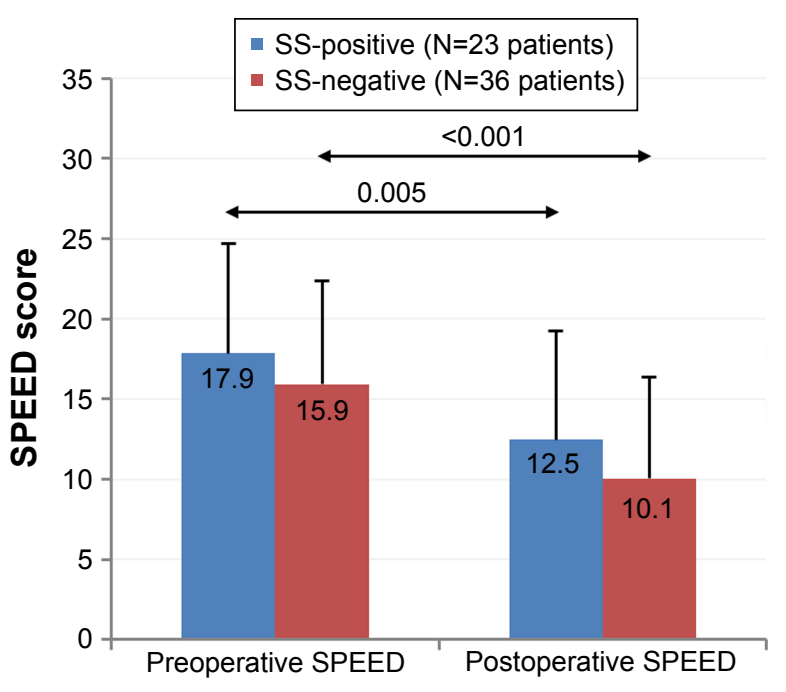

Figure 2 Effect of LipiFlow treatment on Standard Patient Evaluation of Eye Dryness (SPEED) score of Sjögren's syndrome (SS)-positive and SS-negative patients.

post-treatment MGS of SS-negative patients was significantly better than that of SS-positive patients $(P=0.021)$. Likewise, mean SPEED score decreased significantly in both SS-positive $(P=0.005)$ and SS-negative $(P<0.001)$ patients from pretreatment to 8 weeks' post treatment (Figure 2). Post-treatment SPEED scores were not significantly different between the SS-positive and SS-negative patients $(P=0.163)$. Similarly, statistically significant improvement in TBUT was observed from pretreatment level to the post-treatment visit ( 8 weeks) in SS-positive $(P<0.001)$ and SS-negative $(P<0.001)$ patients (Figure 3). Post-LipiFlow TBUT was not significantly different between the SS-positive and SS-negative patients $(P=0.136)$.

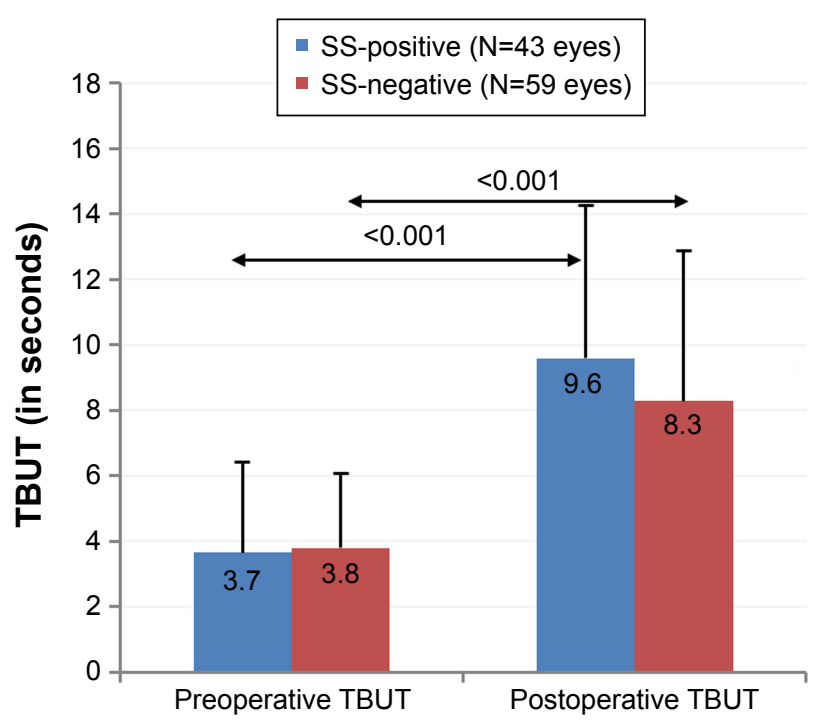

Figure 3 Effect of LipiFlow treatment on tear breakup time (TBUT) of Sjögren's syndrome (SS)-positive and SS-negative patients.

\section{Discussion}

LipiFlow is an automated thermodynamic pulsatile therapeutic procedure, which has been shown to improve meibomian gland function. ${ }^{12,19}$ The single, 12-minute in-office treatment has been found to be highly effective in the treatment of MGD-associated EDE by increasing meibomian gland function, thereby improving TBUT, tear film stability and reducing dry eye symptoms. ${ }^{10-12,14,17-19}$ The results of the procedure in this study were similarly effective in significantly improving the signs (MGS score and TBUT) and symptoms (SPEED score) at 8 weeks' post-treatment as compared to the baseline levels in both SS-positive and SS-negative patients.

SS is an autoimmune disease primarily affecting the exocrine glands, including the lacrimal glands; the resultant effect is a decrease in the production of tears leading to ATDE. ${ }^{1,23}$ Generally, the clinical management of SS-related dry eye remains focused on therapeutic options to address the aqueous component of the tears, such as the instillation of artificial tears, topical cyclosporine A, corticosteroids, punctal occlusion, and so on. ${ }^{24}$ However, a thorough review of literature reveals that SS is frequently associated with MGD. ${ }^{6,7}$ It has been documented previously that the incidence of meibomian gland dropout and the tear evaporation rates is significantly higher in SS-associated ATDE patients than non-SS dry eye patients. ${ }^{6,7}$ This concomitant occurrence of MGD in SS dry eye patients will likely result in the worsening of dry eye symptoms by causing increased tear evaporation of already deficient aqueous component and consequent ocular surface desiccation. ${ }^{7}$

The complementary role of aqueous and lipid components of tear film is most apparent in mixed forms of dry eye; ${ }^{25}$ for example, if excessive evaporation is controlled in eyes with low lacrimal secretions, the eye may be able to maintain a healthier tear osmolarity. ${ }^{26}$ Therefore, in patients with presumed SS and MGD, management of MGD is fundamental in reducing the SS-related dry eye symptoms. Curiously, the detection and treatment of MGD in SS have not been explicitly recommended in the vast majority of even the most recent publications pertaining to the management of SS-associated dry eye..$^{24,27} \mathrm{In}$ a single recent review article on the management of SS patients, Foulks et al mentioned that it is important to evaluate and manage lid margin health (specifically suggesting LipiFlow for MGD) since MGD can and will contribute to symptoms of discomfort in SS patients. ${ }^{28}$ The recommendation for LipiFlow was based on the understanding that the gland contents need to be evacuated in order to rehabilitate gland function. ${ }^{28}$ 
This study demonstrated that LipiFlow treatment was effective in improving the dry eye symptoms not only in SS-negative but also in SS-positive patients with MGD. The mean value of post-treatment SPEED score, TBUT and MGS score improved significantly in both SS-positive and SS-negative patients as compared to the baseline levels. In addition, comparisons between the eyes of SS-positive and SS-negative patients revealed significantly better outcomes in the SS-negative eyes in post-treatment MGS (SS-negative versus SS-positive eyes, $P$-value $=0.021$ ). It is possible that the higher meibomian gland dropout in SS-positive patients, as reported previously by Shimazaki et al, ${ }^{7}$ may be responsible for the comparatively smaller improvement of MGS in SS-positive eyes. However, future studies utilizing meibography are needed to validate this hypothesis.

It is important to note that all patients in this study were already being treated with tear substitutes, nutritional supplements and other therapeutic options for ADDE, but continued to demonstrate signs and symptoms of dry eye disease (high SPEED scores, pretreatment). The LipiFlow treatment was performed as an add-on treatment and the individualized dry eye therapy continued post-LipiFlow as well. A significant improvement in MGS, TBUT and SPEED scores with add-on LipiFlow treatment is in alignment with the current knowledge that to optimize the health of the ocular surface, meibomian gland function must be rehabilitated.

As we have used $\mathrm{Sjö}$ test as the only criterion for the screening of SS, there might be some false-positive and false-negative results of the test, which may have led to some inaccuracies in the analysis. This may be considered as a potential limitation of this study.

Patients with SS should be evaluated for the presence of concomitant MGD, and if present, treatment should be considered. The results of this study indicate that "add-on" LipiFlow treatment in patients who were SS-positive for novel biomarkers of SS and who had concomitant MGD demonstrated significant improvement in signs and symptoms of dry eye, although the improvement in MGS scores of SS-positive patients was lower than that observed in SS-negative patients.

\section{Disclosure}

ATE and RB are consultants to TearScience. CAB is a TearScience employee. KG reports no conflicts of interest in this work.

\section{References}

1. No authors listed. The definition and classification of dry eye disease: report of the definition and classification subcommittee of the international dry eye workshop (2007). Ocul Surf. 2007;5(2):75-92.
2. Coursey TG, de Paiva CS. Managing Sjogren's syndrome and nonSjogren syndrome dry eye with anti-inflammatory therapy. Clin Ophthalmol. 2014;8:1447-1458.

3. Fox RI. Sjogren's syndrome: current therapies remain inadequate for a common disease. Expert Opin Investig Drugs. 2000;9(9):2007-2016.

4. Mavragani CP, Moutsopoulos HM. Conventional therapy of Sjogren's syndrome. Clin Rev Allergy Immunol. 2007;32(3):284-291.

5. Geerling G, Tauber J, Baudouin C, et al. The international workshop on meibomian gland dysfunction: report of the subcommittee on management and treatment of meibomian gland dysfunction. Invest Ophthalmol Vis Sci. 2011;52(4):2050-2064.

6. Goto E, Matsumoto Y, Kamoi M, et al. Tear evaporation rates in Sjogren syndrome and non-Sjogren dry eye patients. Am J Ophthalmol. 2007; 144(1):81-85.

7. Shimazaki J, Goto E, Ono M, Shimmura S, Tsubota K. Meibomian gland dysfunction in patients with Sjogren syndrome. Ophthalmology. 1998;105(8):1485-1488.

8. Suhalim JL, Parfitt GJ, Xie Y, et al. Effect of desiccating stress on mouse meibomian gland function. Ocul Surf. 2014;12(1):59-68.

9. Menzies KL, Srinivasan S, Prokopich CL, Jones L. Infrared imaging of meibomian glands and evaluation of the lipid layer in Sjogren's syndrome patients and nondry eye controls. Invest Ophthalmol Vis Sci. 2015;56(2):836-841.

10. Friedland BR, Fleming CP, Blackie CA, Korb DR. A novel thermodynamic treatment for meibomian gland dysfunction. Curr Eye Res. 2011; 36(2):79-87.

11. Lane SS, DuBiner HB, Epstein RJ, et al. A new system, the LipiFlow, for the treatment of meibomian gland dysfunction. Cornea. 2012;31(4):396-404.

12. Blackie CA, Carlson AN, Korb DR. Treatment for meibomian gland dysfunction and dry eye symptoms with a single-dose vectored thermal pulsation: a review. Curr Opin Ophthalmol. 2015;26(4):306-313.

13. Gifford S. Meibomian glands in chronic blepharoconjunctivitis. Am J Ophthalmol. 1921;4(7):489-494.

14. Greiner JV. A single LipiFlow ${ }^{\circledR}$ thermal pulsation system treatment improves meibomian gland function and reduces dry eye symptoms for 9 months. Curr Eye Res. 2012;37(4):272-278.

15. Qiao J, Yan X. Emerging treatment options for meibomian gland dysfunction. Clin Ophthalmol. 2013;7:1797-1803.

16. Finis D, Hayajneh J, Konig C, Borrelli M, Schrader S, Geerling G. Evaluation of an automated thermodynamic treatment $\left(\right.$ LipiFlow $\left.^{\circledR}\right)$ system for meibomian gland dysfunction: a prospective, randomized, observer-masked trial. Ocul Surf. 2014;12(2):146-154.

17. Finis D, Konig C, Hayajneh J, Borrelli M, Schrader S, Geerling G. Six-month effects of a thermodynamic treatment for MGD and implications of meibomian gland atrophy. Cornea. 2014;33(12):1265-1270.

18. Greiner JV. Long-term (12-month) improvement in meibomian gland function and reduced dry eye symptoms with a single thermal pulsation treatment. Clin Exp Ophthalmol. 2013;41(6):524-530.

19. Korb DR, Blackie CA. Restoration of meibomian gland functionality with novel thermodynamic treatment device-a case report. Cornea. 2010;29(8):930-933.

20. Galor A. MGD: definition versus dry eye disease, risk factors. Curr Ophthalmol Rep. 2014;2(2):58-64.

21. Beckman KA. Detection of early markers for Sjogren syndrome in dry eye patients. Cornea. 2014;33(12):1262-1264.

22. Ngo W, Situ P, Keir N, Korb D, Blackie C, Simpson T. Psychometric properties and validation of the standard patient evaluation of eye dryness questionnaire. Cornea. 2013;32(9):1204-1210.

23. Jung HH, Ji YS, Sung MS, Kim KK, Yoon KC. Long-term outcome of treatment with topical corticosteroids for severe dry eye associated with Sjogren's syndrome. Chonnam Med J. 2015;51(1):26-32.

24. Maslinska M, Przygodzka M, Kwiatkowska B, Sikorska-Siudek K. Sjogren's syndrome: still not fully understood disease. Rheumatol Int. 2015;35(2):233-241.

25. Blackie CA, Korb DR, Knop E, Bedi R, Knop N, Holland EJ. Nonobvious obstructive meibomian gland dysfunction. Cornea. 2010;29(12): $1333-1345$. 
26. Mathers WD. Ocular evaporation in meibomian gland dysfunction and dry eye. Ophthalmology. 1993;100(3):347-351.

27. Vitali C, Palombi G, Cataleta P. Treating Sjogren's syndrome: insights for the clinician. Ther Adv Musculoskelet Dis. 2010;2(3):155-166.
28. Foulks GN, Forstot SL, Donshik PC, et al. Clinical guidelines for management of dry eye associated with Sjogren disease. Ocul Surf. 2015; 13(2):118-132.

\section{Publish your work in this journal}

Clinical Ophthalmology is an international, peer-reviewed journal covering all subspecialties within ophthalmology. Key topics include: Optometry; Visual science; Pharmacology and drug therapy in eye diseases; Basic Sciences; Primary and Secondary eye care; Patient Safety and Quality of Care Improvements. This journal is indexed on

\section{Dovepress}

PubMed Central and CAS, and is the official journal of The Society of Clinical Ophthalmology (SCO). The manuscript management system is completely online and includes a very quick and fair peer-review system, which is all easy to use. Visit http://www.dovepress.com/ testimonials.php to read real quotes from published authors. 